\title{
Thermal leptogenesis in a model with mass varying neutrinos
}

\author{
Xiao-Jun Bi, ${ }^{1}$ Peihong Gu, ${ }^{1}$ Xiulian Wang, ${ }^{2}$ and Xinmin Zhang ${ }^{1}$ \\ 1 Institute of High Energy Physics, \\ Chinese Academy of Sciences, P.O. Box 918-4, \\ Beijing 100039, People's Republic of China \\ ${ }^{2}$ Institute of theoretical Physics, Chinese Academy of Sciences, \\ P.O. Box 2735, Beijing 100080, People's Republic of China
}

(Dated: October 17, 2018)

\begin{abstract}
In this paper we consider the possibility of neutrino mass varying during the evolution of the Universe and study its implications on leptogenesis. Specifically, we take the minimal seesaw model of neutrino masses and introduce a coupling between the right-handed neutrinos and the dark energy scalar field, the Quintessence. In our model, the right-handed neutrino masses change as the Quintessence scalar evolves. We then examine in detail the parameter space of this model allowed by the observed baryon number asymmetry. Our results show that it is possible to lower the reheating temperature in this scenario in comparison with the case that the neutrino masses are unchanged, which helps solve the gravitino problem. Furthermore, a degenerate neutrino mass patten with $m_{i}$ larger than the upper limit given in the minimal leptogenesis scenario is permitted.
\end{abstract}




\section{INTRODUCTION}

The baryon number asymmetry of the Universe has been determined to the precision of less than $10 \%$ after the first year observations of the Wilkinson Microwave Anisotropy Probe (WMAP) experiment [1]. The reported value of the asymmetry is

$$
\eta_{B} \equiv \frac{n_{B}}{n_{\gamma}}=6.1_{-0.2}^{+0.3} \times 10^{-10}
$$

where $n_{B}=n_{b}-n_{\bar{b}}$ and $n_{\gamma}$ are the baryon and photon number densities, respectively.

On the theoretical side, many models have been proposed in the literature[2] to explain the small while non-zero $\eta_{B}$ dynamically. Among various models, leptogenesis is one of the most attractive scenarios [3], where lepton number asymmetry is converted to baryon number asymmetry via the $(B+L)$-violating sphaleron interactions [4]. This mechanism has been studied extensively in the literature [5, 6, 7, 8, 9, 10].

In the minimal scenario of leptogenesis, the standard model is extended by including three generations of right-handed $(\mathrm{RH})$ neutrinos, which are Majorana fermions and couple to the lepton doublets through Yukawa couplings. The heavy RH neutrinos are produced in the early universe by thermal scattering with the primordial thermal bath via the Yukawa coupling. In this framework, the cosmological baryon asymmetry is naturally connected to the neutrino properties through the seesaw mechanism[11], which is the most natural explanation for the tiny neutrino masses observed in the neutrino oscillation experiments [12]. As shown in Ref. 8], the amount of the baryon asymmetry generated in this minimal thermal leptogenesis scenario can be characterized by four parameters: the maximal CP asymmetry $\epsilon_{1}$, the lightest RH neutrino mass $M_{1}$, the effective light neutrino mass $\widetilde{m}$ and the quadratic mean of the light neutrino masses $\bar{m}$, under the assumption that the dominant contribution to the lepton asymmetry is given by the decay of the lightest RH neutrino, $N_{1}$. The detailed calculations show that to produce the observed baryon asymmetry, it requires $M_{1} \gtrsim 10^{10} \mathrm{GeV}$ and $\bar{m} \lesssim 0.2 \mathrm{eV}$ which corresponds to $m_{i} \lesssim 0.12 \mathrm{eV}$.

Although it is theoretically elegant and simple, this scenario seems to need a high reheating temperature, $T_{R} \sim M_{1} \gtrsim \mathcal{O}\left(10^{10} \mathrm{GeV}\right)$, which is only marginally compatible with the bound from the gravitino problem, $T_{R} \leq\left(10^{8}-10^{10}\right) \mathrm{GeV}$, in supersymmetric models [13]. The degenerate solution of the neutrino masses is also strongly disfavored in this scenario.

Note that if the evidence of neutrinoless double $\beta$ decay with $m_{e e}=(0.05-0.86) \mathrm{eV}$ [14] (at 
95\% C.L.) is confirmed, a degenerate neutrino spectrum is required. A recent study on the cosmological data also showed a preference for degenerate neutrinos with $m_{i}=0.2 \mathrm{eV}[15]$. A possible way to accommodate the degenerate neutrinos by the thermal leptogenesis is the resonant enhancement of the $\mathrm{CP}$ asymmetry when the heavy $\mathrm{RH}$ neutrinos are nearly degenerate, $\left|M_{2,3}-M_{1}\right|=\mathcal{O}\left(\Gamma_{i}\right)[\underline{6}, \underline{9}]$.

In the standard $\Lambda \mathrm{CDM}$ cosmology, the dark energy which drives the universe accelerating weights about $73 \%$ of the total energy of the universe 1, 16, 17]. However, the nature of the dark energy (DE) remains mysterious. It could be simply a remnant small cosmological constant. However, many physicists are attracted by the dynamical solution with a scalar field (or multi-scalar fields) like Quintessence. Being a dynamical component, the scalar field dark energy is expected to interact with the ordinary matter. In the literature there have been a lot of studies on the possible couplings of Quintessence to baryon, dark matter and photons [18]. Recent data on the possible variation of the electromagnetic fine structure constant reported in 19] has triggered interests in studies related to the interactions between Quintessence and the matter fields.

Recently we have studied various possible interactions, with or without supersymmetry, between Quintessence and the matter field of the electroweak standard model [20]. In a previous paper 21] we considered a possibility of neutrinos coupling with Quintessence and studied its implications on cosmology. In this paper we propose a scenario that the righthanded neutrinos in the minimal seesaw model couple to Quintessence and examine its implications on the thermal leptogenesis. Other possible implications of varying neutrino masses in astrophysics and cosmology are discussed in Ref. [22]. With a detailed numerical calculation we will show that in this scenario the reheating temperature can be as low as about $10^{8} \mathrm{GeV}$ and the degenerate neutrino mass patten with $m_{i} \sim \mathcal{O}(0.2 \mathrm{eV})$ will be allowed.

This paper is organized as follows. In Sec II, we will present the framework and formulation for the calculation of leptogenesis in our model. We will explain how the interaction between the right-handed neutrinos and Quintessence affect $\eta_{B}$ in terms of the four parameters $\epsilon_{1}, M_{1}, \widetilde{m}$ and $\bar{m}$. In section III, we will present the numerical results. In section IV, we consider a Quintessence model and propose a specific example of Quintessence to RH neutrinos, then study the interplay between the leptogenesis and the evolution of Quintessence. Sec. $\mathrm{V}$ is the summary and conclusion of this paper. 


\section{INTERACTING DARK ENERGY SCALAR FIELD WITH NEUTRINOS}

\section{A. The model}

We extend the minimal seesaw model by introducing a new coupling between Quintessence scalar field, $Q$, and the $\mathrm{RH}$ neutrinos. The Lagrangian relevant to leptogenesis is given by

$$
\mathcal{L}=\mathcal{L}_{\text {lep }}+\mathcal{L}_{Q}
$$

with

$$
-\mathcal{L}_{\text {lep }}=Y_{i j} \bar{L}_{i} \tilde{H} N_{j}+\frac{1}{2} M_{i}(Q) N_{i}^{T} C N_{i}+\text { h.c. }
$$

and

$$
\mathcal{L}_{Q}=\frac{1}{2} \partial_{\mu} Q \partial^{\mu} Q-V(Q),
$$

where $Y_{i j}$ is the Yukawa coupling of the $\mathrm{RH}$ neutrinos, $M_{i}(Q)$ is the Majorana masses of the RH neutrinos which is now a function of the value of $Q$, and $V(Q)$ is the potential of the Quintessence. We have taken the basis that the RH Majorana mass matrix is diagonal. The form of $V(Q)$ for a specific model and an explicit form of $M(Q)$ will be given in Sec. IV. The Quintessence dependent masses of the right-handed neutrinos give rise to different Majorana masses at the early epoch from that at the present epoch, which is the key for the difference of our model from the usual leptogenesis scenario.

\section{B. The CP asymmetry}

Generally, the Yukawa coupling $Y$ contains CP violating phases and lead to different branching ratios for $N$ decays into lepton and antilepton. The asymmetry for $N_{1}$ decays is $[5,6]$

$$
\begin{aligned}
\epsilon_{1} & =\frac{\Gamma\left(N_{1} \rightarrow L H\right)-\Gamma\left(N_{1} \rightarrow \bar{L} \bar{H}\right)}{\Gamma\left(N_{1} \rightarrow L H\right)+\Gamma\left(N_{1} \rightarrow \bar{L} \bar{H}\right)} \\
& \approx-\frac{3}{16 \pi} \frac{1}{\left(Y^{\dagger} Y\right)_{11}} \sum_{i=2,3} \operatorname{Im}\left[\left(Y^{\dagger} Y\right)_{1 i}\right]^{2} \frac{M_{1}}{M_{i}}, \text { for } M_{1} \ll M_{2}, M_{3} .
\end{aligned}
$$

Since the light neutrino masses are generated by the seesaw mechanism[11] and constrained by the neutrino oscillation experiments, the CP asymmetry is also constrained by the neutrino data. In the case of hierarchical neutrinos, i.e., $m_{3} \approx \sqrt{\Delta m_{\text {atm }}^{2}} \approx 0.05 \mathrm{eV} \gg$ 
$\sqrt{\Delta m_{\text {sol }}^{2}} \sim 0.008 \mathrm{eV} \gg m_{1}$, an approximate upper bound on the CP asymmetry [7] is

$$
\left|\epsilon_{1}\right| \lesssim \frac{3}{16 \pi} \frac{M_{1} \sqrt{\Delta m_{a t m}^{2}}}{v^{2}}
$$

with $v=174 G e V$ being the vacuum expectation value of the neutral Higgs boson. For the degenerate case, i.e., $m_{1} \approx m_{2} \approx m_{3} \approx \bar{m} / \sqrt{3} \gg \sqrt{\Delta m_{a t m}^{2}}$, the upper bound is given by

$$
\left|\epsilon_{1}\right| \lesssim \frac{3 \sqrt{3}}{16 \pi} \frac{M_{1} \Delta m_{a t m}^{2}}{v^{2} \bar{m}}
$$

where $\bar{m}=\sqrt{m_{1}^{2}+m_{2}^{2}+m_{3}^{2}}$ is the quadratic mean of the neutrino masses. One can see that the maximal $\epsilon_{1}$ is proportional to $M_{1}$ while inversely proportional to $\bar{m}$. In the following we always take the upper bound of $\epsilon_{1}$ to study the maximal value of $\eta_{B}$ on the parameter space.

The final lepton asymmetry is determined in general by

$$
Y_{L} \equiv \frac{n_{L}-n_{\bar{L}}}{s}=\kappa \frac{\epsilon_{1}}{g_{*}}
$$

where $s$ is the entropy density, $g_{*}$ represents the number of the relativistic degrees of freedom at the time when $N_{1}$ decays and $\kappa<1$ represents the washout effects for the lepton number asymmetry in the thermal bath. The lepton asymmetry in Eq. (8) will be converted partly into baryon asymmetry by the electroweak sphaleron process taking into account the gauge, Yukawa interaction and the QCD sphaleron effects[23], $Y_{B}=a Y_{B-L} \approx-a Y_{L}$, with $a=$ $28 / 79[10,24]$.

\section{The washout effect}

The factor $\kappa$ in Eq. (8) can be calculated by solving the Boltzmann equations [8, 10]. At the leading order of $\epsilon_{1}$ one has the Boltzmann equations for $Y_{N_{1}}=n_{N_{1}} / s$ and $Y_{B-L}=$ $n_{B-L} / s$,

$$
\begin{aligned}
\frac{s H}{z} \frac{d Y_{N_{1}}}{d z} & =-\left(\frac{Y_{N_{1}}}{Y_{N_{1}}^{e q}}-1\right)\left(\gamma_{D}+2 \gamma_{H, s}+4 \gamma_{H, t}\right), \\
\frac{s H}{z} \frac{d Y_{B-L}}{d z} & =-\epsilon_{1} \gamma_{D}\left(\frac{Y_{N_{1}}}{Y_{N_{1}}^{e q}}-1\right)-\frac{Y_{B-L}}{Y_{L}^{e q}} \gamma_{W},
\end{aligned}
$$

with

$$
\gamma_{W}=\frac{1}{2} \gamma_{D}+2 \gamma_{N}+2 \gamma_{N, t}+2 \gamma_{H, t}+\frac{Y_{N_{1}}}{Y_{N_{1}}^{e q}} \gamma_{H, s}
$$


where $\gamma$ is the space time density of the scatterings in thermal equilibrium. In the equations above, $\gamma_{D}$ refers to the reaction density for the process of $N_{1}$ decays (and inverse decays), $N_{1} \leftrightarrow L H(\bar{L} \bar{H}), \gamma_{H, s}$ and $\gamma_{H, t}$ for the processes of $\Delta L=1$ scatterings via exchanging Higgs boson, $N_{1} L(\bar{L}) \leftrightarrow \bar{t}(t) Q(\bar{Q})$ in $s$ channel and $N_{1} t(\bar{t}) \leftrightarrow \bar{L}(L) Q(\bar{Q})$ in $t$ channel, $\gamma_{N}$ and $\gamma_{N, t}$ for the processes of $\Delta L=2$ scatterings via exchanging the $\mathrm{RH}$ neutrinos, $L H \leftrightarrow \bar{L} \bar{H}$ in both $s$ and $t$ channels and $L L \leftrightarrow \bar{H} \bar{H}, \bar{L} \bar{L} \leftrightarrow H H$ in $t$ channel.

In Eq. (9), the minus sign on the right-hand side means that all the reactions drive the $Y_{N_{1}}$ to its equilibrium value $Y_{N_{1}}^{e q}$. In Eq. (10), the first term on the right-hand side is the source term, which gives rise to non-zero $Y_{B-L}$, while the second term represents washout effects that lead $Y_{B-L}$ to zero.

The $\gamma_{N}$ consists two parts, the resonant part, $\gamma_{N, r e s}$ and the non-resonant part, $\gamma_{N, n o n}$, i.e., $\gamma_{N}=\gamma_{N, r e s}+\gamma_{N, \text { non }}$. The $\gamma_{D}, \gamma_{H, s}, \gamma_{H, t}$ and $\gamma_{N, \text { res }}$ are all proportional to the effective neutrino mass $[8]$

$$
\widetilde{m}=\frac{\left(Y^{\dagger} Y\right)_{11} v^{2}}{M_{1}}
$$

while $\gamma_{N, \text { non }}$ and $\gamma_{N, t}$ are proportional to $M_{1} \bar{m}^{2}$. These properties are essential in understanding the behavior of the solutions of the Boltzmann equations. When $\delta \gamma_{W}=\gamma_{N, n o n}+\gamma_{N, t} \propto$ $M_{1} \bar{m}^{2}$ is negligible, i.e., at $M_{1}(\bar{m} / 0.1 e V)^{2}<10^{13} \mathrm{GeV}[8]$, the washout factor $\kappa$ is mainly determined by $\widetilde{m}$ and nearly independent of $M_{1}$ and $\bar{m}$. The effect of $\widetilde{m}$ has two different aspects. When it is very small $\left(\lesssim 10^{-3} \mathrm{eV}\right)$, the RH neutrinos will not be able to reach thermal equilibrium via the scattering with the thermal bath, so $\kappa$ will be small. Conversely, if $\widetilde{m}$ is too large the $\mathrm{RH}$ neutrinos can be brought into thermal equilibrium rapidly. However, the washout effect will also be large, consequently we have a small $\kappa$. Around $\widetilde{m} \sim 10^{-3} \mathrm{eV}$, $\kappa$ reaches its maximal value. When $\delta \gamma_{W}$ is sizable $\kappa$ will be suppressed exponentially, since the washout effect is given by

$$
\kappa(z)=\int_{z_{i n}}^{z} d z^{\prime} \gamma_{D}\left(z^{\prime}\right) \Delta\left(z^{\prime}\right) e^{-\int_{z^{\prime}}^{z} d z^{\prime \prime} z^{\prime \prime} \gamma_{W}\left(z^{\prime \prime}\right) /\left(s H Y_{L}^{e q}\right)},
$$

with $\Delta(z)=\left(Y_{N_{1}} / Y_{N_{1}}^{e q}-1\right) z /(s H)$. In this case, $\kappa$ becomes nearly independent of $\widetilde{m}$.

In short, $\epsilon_{1}$ (as well as $Y_{L}$ ) increases with $M_{1}$ while decreases with $\bar{m}$ linearly when $\delta \gamma_{W}$ is negligible, $M_{1}(\bar{m} / 0.1 e V)^{2}<10^{13} G e V[8]$. However, when $\delta \gamma_{W} \propto M_{1} \bar{m}^{2}$ is sizable, $M_{1}(\bar{m} / 0.1 e V)^{2}>10^{13} \mathrm{GeV}$, the final asymmetry $Y_{L}$ will be suppressed exponentially [8]. The $\kappa$ reaches maximal at $\widetilde{m} \sim 10^{-3} \mathrm{eV}$ when the effect of $\delta \gamma_{W}$ is negligible. It is shown in Ref. 
8] that to generate the required value of the baryon number asymmetry, $\eta_{B}$, one needs $M_{1} \gtrsim 10^{10} \mathrm{GeV}$ and $\bar{m} \lesssim 0.2 \mathrm{eV}$ in general.

\section{The effect of Quintessence}

With the interaction between the Quintessence and the $\mathrm{RH}$ neutrinos, $M_{1}(Q)$ is now a function of the Quintessence field. $M_{1}$ at the epoch when it decays will differ from that at the present epoch. We introduce a parameter $K$

$$
K\left(Q^{D}\right)=\frac{M_{1}^{0}}{M_{1}^{D}}
$$

where $M_{1}^{0}=M_{1}\left(Q^{0}\right)$ and $M_{1}^{D}=M_{1}\left(Q^{D}\right)$ denote the Majorana masses of the $\mathrm{RH}$ neutrinos at present and at the decay time. For simplicity we take $K$, as well as $M_{1}$, as a constant when solving the Boltzmann equations. In section IV, we will show that $Q$ is quite flat during the period of leptogenesis for the model we consider and this is a reasonable assumption. We then have

$$
m_{\nu}^{D}=-Y \frac{1}{M^{D}} Y^{T} v^{2}=m_{\nu}^{0} \cdot K, \bar{m}^{D}=\bar{m}^{0} \cdot K, \widetilde{m}^{D}=\widetilde{m}^{0} \cdot K
$$

where the masses with the superscripts " $\mathrm{D}$ " and " 0 " correspond to the values evaluated at the leptogenesis and at the present time respectively.

\section{NUMERICAL RESULTS}

In this section we present our numerical results by solving the Boltzmann equations. Besides $M_{1}, \bar{m}$ and $\widetilde{m}$ we now introduce a new parameter $K$. We will show that varying $K$ makes it possible for $M_{1}$ to be as low as about $10^{8} \mathrm{GeV}$ and $\bar{m}$ can be as large as $0.4 \mathrm{eV}$ which corresponds to $\left.m_{i} \approx 0.23 e V[1]\right]^{1}$.

In Fig. 1, we plot the baryon number asymmetry $\eta_{B}$ as a function of $K$ for the hierarchical neutrino mass spectrum, i.e., $\bar{m}^{0}=0.05 \mathrm{eV}$. On the left panel, we take $M_{1}^{D}=10^{8} \mathrm{GeV}$ for $\widetilde{m}^{0}=10^{-4}, 10^{-5}, 10^{-6} \mathrm{eV}$ respectively. One can see from the figure that $\eta_{B}$ increases

$\overline{1}$ This is the upper bound set by WMAP. Recent SDSS 17 lowers this limit to $0.2 \mathrm{eV}$. When neutrino masses vary during the evolution of the Universe, however, these cosmological bounds on the absolute neutrino masses may be different which is worthwhile studying further. 

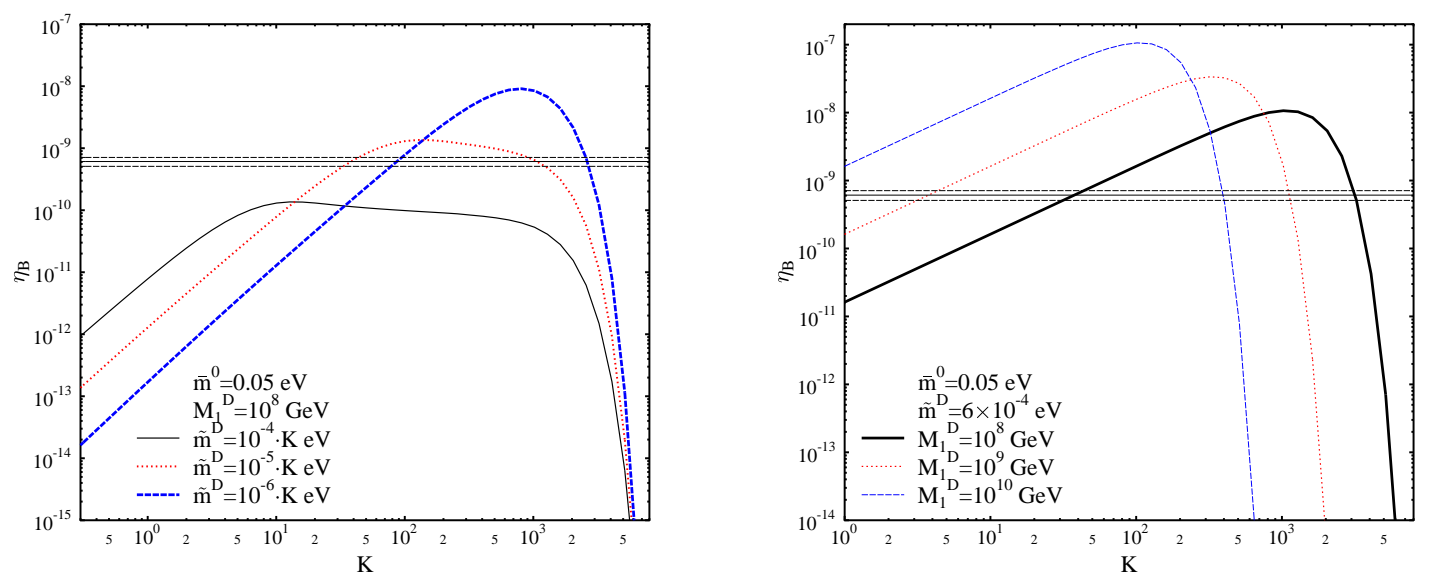

FIG. 1: The baryon number asymmetry $\eta_{B}$ as a function of $K$ for the hierarchical neutrino mass spectrum, i.e., $\bar{m}^{0}=0.05 \mathrm{eV}$. On the left panel, we take $M_{1}^{D}=10^{8} \mathrm{GeV}$ and $\widetilde{m}^{0}=10^{-4}, 10^{-5}, 10^{-6} \mathrm{eV}$ respectively. On the right panel, we take $\widetilde{m}^{D}=6 \times 10^{-4} \mathrm{eV}$ and $M_{1}^{D}=10^{8}, 10^{9}, 10^{10} \mathrm{GeV}$ respectively. The horizontal lines represent $\eta_{B}=5.1,6.1,7.1 \times 10^{-10}$ respectively.

with $K$ linearly until $\widetilde{m}^{D} \approx 10^{-3} \mathrm{eV}$, i.e., $\eta_{B}$ increases with $K$ until $K \approx 10^{1}, 10^{2}, 10^{3}$ for $\widetilde{m}^{0}=10^{-4}, 10^{-5}, 10^{-6} \mathrm{eV}$ respectively. In these regions, the amount of enhancement of $\epsilon_{1}$ as $K$ gets large dominates over that of the washout effect so that $\eta_{B}$ increases linearly with $K$. However, as the $\bar{m}^{D}$ is about $\bar{m}^{0} \cdot K \sim 0.05 \cdot 10^{3} \mathrm{eV}, \delta \gamma_{W}$ becomes sizable then $\eta_{B}$ decreases exponentially as $K$. In the region between these two points, $\eta_{B}$ is a flat function of $K$. This is due to the effect of $K$ which enhances $\epsilon_{1}$ on one hand while decrease $\kappa$ as $\widetilde{m}^{D} \gtrsim 10^{-3} \mathrm{eV}$ on the other hand. On the right panel, we fix $\widetilde{m}^{D}=6 \times 10^{-4} \mathrm{eV}$, which gives the maximal $\kappa$ for the negligible $\delta \gamma_{W}\left[\underline{8]}\right.$, and take $M_{1}^{D}=10^{8}, 10^{9}, 10^{10} \mathrm{GeV}$ respectively. One can see from this figure that $\eta_{B}$ increases with $K$ linearly until $M_{1}^{D}\left(\bar{m}^{D} / 0.1 \mathrm{eV}\right)^{2} \sim 10^{13} \mathrm{GeV}$. The different behavior between the two figures is easy to understand if one notices that $\widetilde{m}^{D}$ is fixed and does not increase with $K$ on the right panel.

In Fig. 2, we plot the contour for $\eta_{B}=5.5 \times 10^{-10}$ in the $M_{1}^{D}-K$ plane for the hierarchical neutrinos. One can see from this figure that as $\mathrm{K}$ gets large, $M_{1}^{D}$ is allowed to take a smaller value. For example, at $K \approx 30, M_{1}^{D}$ can be as low as about $10^{8} \mathrm{GeV}$. As a result of it, the reheating temperature required will be lowered than that in the minimal thermal leptogenesis.

In Fig. 3, we plot $\eta_{B}$ as a function of $K$ for the degenerate neutrinos, taking $\bar{m}^{0}=0.4 \mathrm{eV}$ 


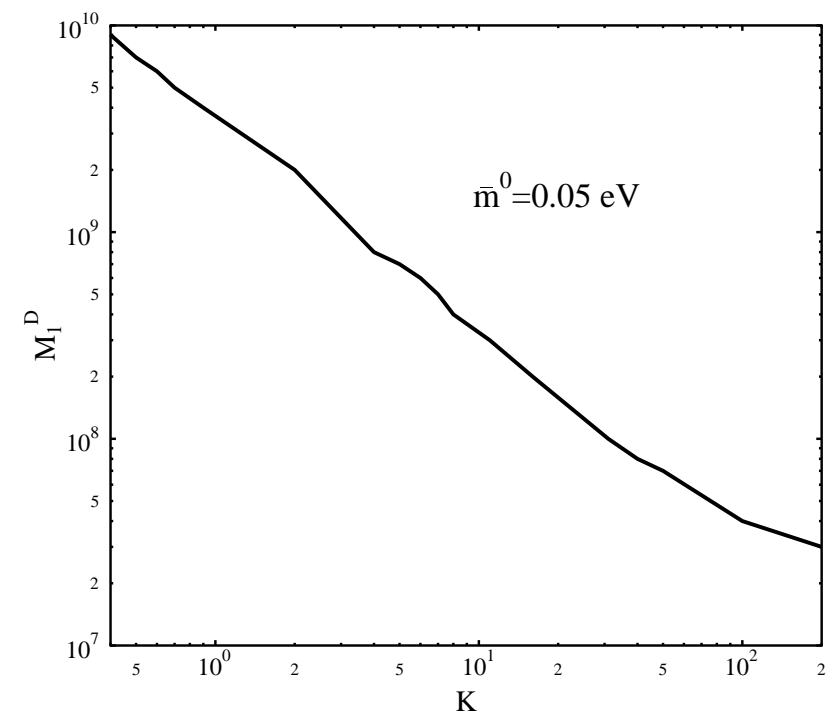

FIG. 2: The contour of $\eta_{B}=5.5 \times 10^{-10}$ in the $M_{1}^{D}-K$ plane for the hierarchical neutrino spectrum, $\bar{m}^{0}=0.05 \mathrm{eV}$.

$\left(m_{i} \approx 0.23 \mathrm{eV}\right)$ for an example. It should be noticed that $\widetilde{m}$ is constrained as $m_{1}<\widetilde{m}<m_{3}$ if no fine tunning is taken [8]. We take $\widetilde{m}^{0} \approx m_{i}^{0} \approx 0.23 \mathrm{eV}$ in this case. The figure looks similar to that for the hierarchical case. For $\widetilde{m}^{D}=(0.23 \cdot \mathrm{K}) \mathrm{eV} \lesssim 10^{-3} \mathrm{eV}, \eta_{B}$ increases linearly with $K$. At the point $M_{1}^{D}\left(\bar{m}^{D} / 0.1 e V\right)^{2} \sim 10^{13} \mathrm{GeV}, \eta_{B}$ starts to decrease exponentially as $K$. In the region between, $\eta_{B}$ is almost a flat function of $K$. We notice that for $K=1$, which corresponds to the case of vanishing Quintessence coupling, no parameter space will satisfy the observed $\eta_{B}$. However, for $M_{1}^{D}=10^{13} \mathrm{GeV}, \eta_{B}$ can meet the requirement for $K \lesssim 0.5$, which is not far from $K=1$. One can see that non-zero $K$ makes it possible to generate $\eta_{B}$ required for the degenerate neutrino mass spectrum. However, in this case we have to take $K$ to be smaller than 1 so that $\kappa$ is not suppressed exponentially. This leads to a small $\epsilon_{1}$ too, which forces us to take a bigger $M_{1}^{D}$. Therefore, it will not be possible to lower $M_{1}^{D}$ and increase $\bar{m}^{0}$ simultaneously.

In Fig. 4 we plot the contour of $\eta_{B}=5.5 \times 10^{-10}$ in the $M_{1}^{D}-K$ plane for the degenerate neutrinos. One can see that $M_{1}^{D}$ decreases linearly firstly, then starts to increases slowly. At $K \approx 0.007, M_{1}^{D}$ reaches its minimal value, $M_{1}^{D} \approx 4.5 \times 10^{12} \mathrm{GeV}$. 


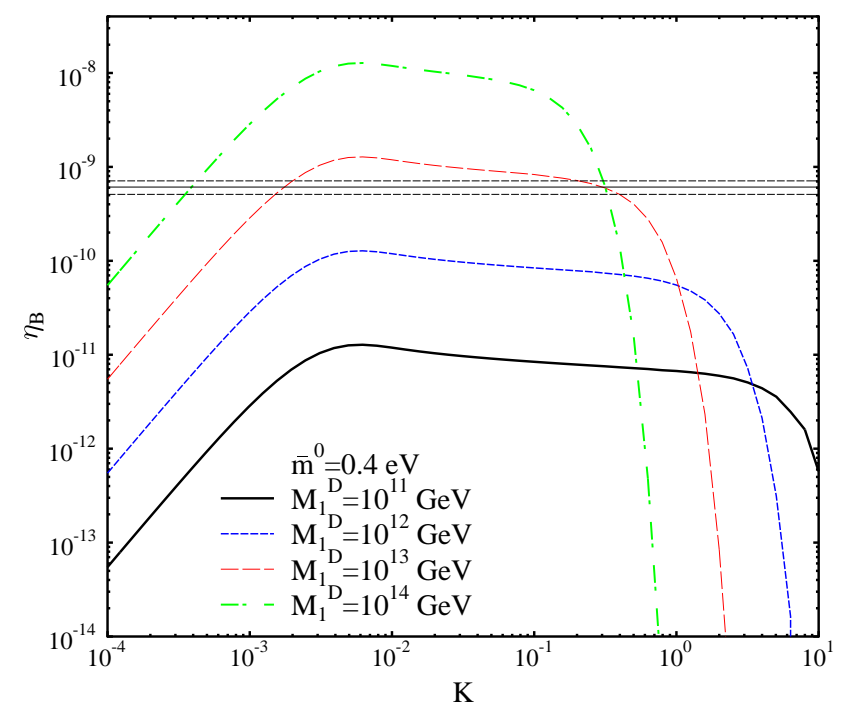

FIG. 3: The baryon number asymmetry $\eta_{B}$ as a function of $K$ for the degenerate neutrino mass spectrum, $\bar{m}^{0}=0.4 \mathrm{eV}$. We take $M_{1}^{D}=10^{11}, 10^{12}, 10^{13}, 10^{14} \mathrm{GeV}$ respectively. The horizontal lines represent $\eta_{B}=5.1,6.1,7.1 \times 10^{-10}$ respectively.

\section{QUINTESSENCE MODELS}

In the last section we present our numerical results of leptogenesis taking $K$ as a free parameter. In this section we will consider a specific Quintessence model and propose a specific form of its coupling to the RH neutrinos. We will study numerically the evolution of $Q$ taking into account the back reaction of the neutrino background and calculate the factor $K$.

In the literature, there have been various proposals for the explicit form of couplings in studying the interaction between the Quintessence and the matter field. For example, one usually introduces $Q F_{\mu \nu} F^{\mu \nu}$ ( $F_{\mu \nu}$ is the electromagnetic field strength tensor) to study the variation of the electromagnetic fine structure constant. However, in an attempt to understand the puzzle why the density of dark matter and dark energy are nearly equal today, the authors of Ref. [25] recently consider a model of interacting Quintessence with dark matter and in their scenario the mass of dark matter particle depends exponentially on the value of $Q, m(Q)=\bar{m} e^{-\lambda Q / M_{p l}}$.

In this paper, we assume that the coupling between Quintessence and the RH neutrinos 


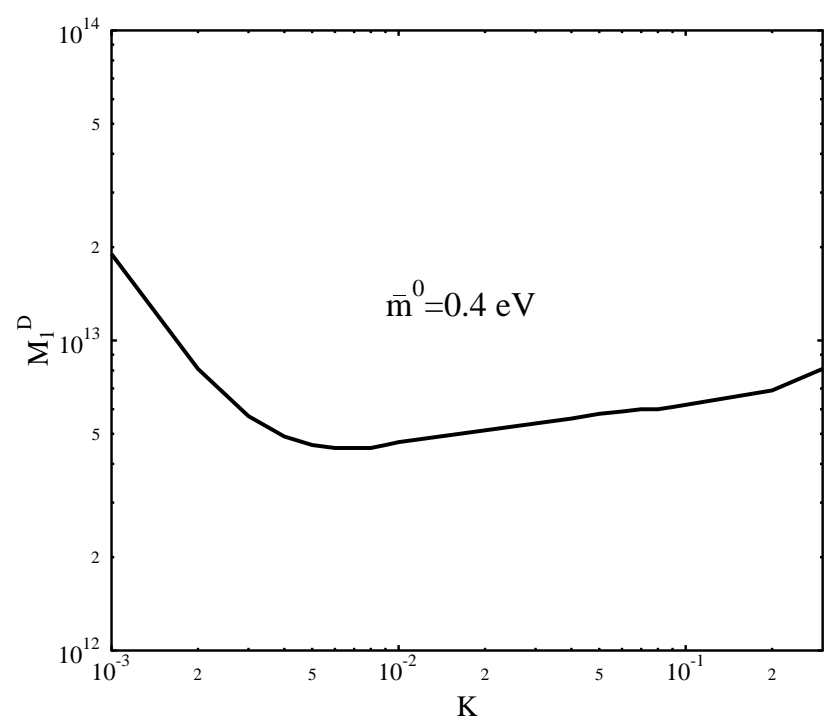

FIG. 4: The contour of $\eta_{B}=5.5 \times 10^{-10}$ in the $M_{1}^{D}-K$ plane for the degenerate neutrino spectrum, $\bar{m}^{0}=0.4 \mathrm{eV}$.

takes a simple form as

$$
M_{i}(Q)=\bar{M}_{i} e^{\beta \frac{Q}{M_{p l}}},
$$

where $\beta$ is a $\mathcal{O}(1)$ coefficient. The ratio $K$ is then given as

$$
K \equiv \frac{M_{i}^{0}}{M_{i}^{D}}=e^{\beta \frac{Q^{0}-Q^{D}}{M_{p l}}}
$$

For a numerical calculation of $K$, we consider a Quintessence model with the double exponential potential [26]

$$
V=V_{0}\left(e^{\lambda Q}+e^{\alpha Q}\right)
$$

This model has the tracking property for suitable parameters. In the absence of coupling in Eq. (16), the evolution of Quintessence is described by the equations

$$
\begin{aligned}
& H^{2}=\frac{8 \pi G}{3}\left(\rho_{m}+\rho_{\gamma}+\rho_{Q}\right), \\
& \ddot{Q}+3 H \dot{Q}+\frac{d V(Q)}{d Q}=0,
\end{aligned}
$$

where $H$ is the Hubble constant, $\rho_{m}, \rho_{\gamma}$ and $\rho_{Q}$ represent the energy densities of matter, radiation and Quintessence respectively. We choose the model parameters as $\lambda=100 M_{p l}^{-1}$, $\alpha=-100 M_{p l}^{-1}$, the initial value of Quintessence field $Q_{i}=1.374 M_{p l}$ and for the state of 

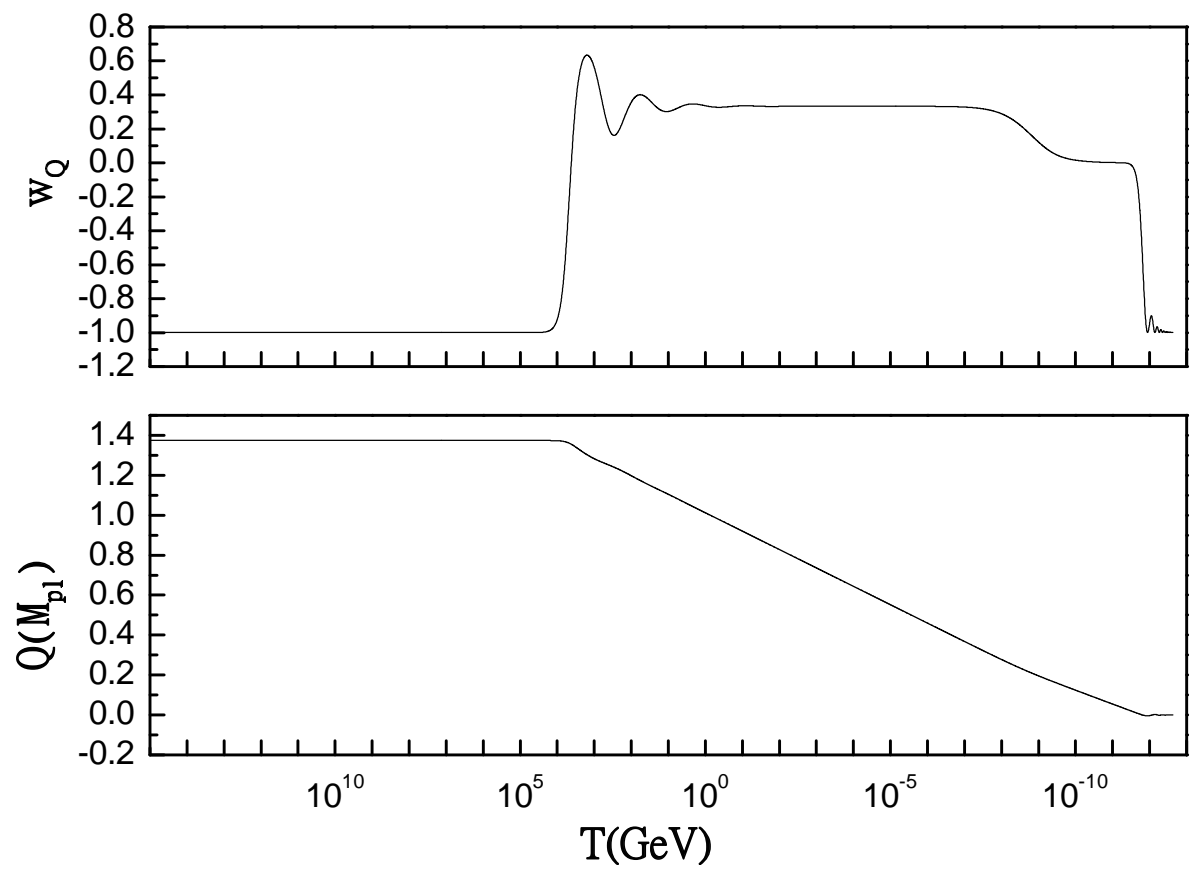

FIG. 5: The evolution of $W_{Q}$ and $Q$ as a function of the temperature $T$ for the double exponential Quintessence model without the coupling with the RH neutrinos.

equation, which is defined as

$$
W_{Q}=\frac{p_{Q}}{\rho_{Q}}=\frac{\dot{Q}^{2} / 2-V(Q)}{\dot{Q}^{2} / 2+V(Q)}
$$

the initial value is $W_{Q_{i}}=-1$. We obtain that $\Omega_{Q_{0}} \simeq 0.72$ and the present equation of state of Quintessence is $W_{Q_{0}} \approx-1$ which are consistent with the observational data. In Fig. 5 we show the evolution of $W_{Q}$ and $Q$ with the temperature $T$. One can see that Quintessence begins to track the background at $T \lesssim 10^{4} \mathrm{GeV}$.

Taking into account the interaction with the $\mathrm{RH}$ neutrinos, we get the equation of evolution of Quintessence as

$$
\ddot{Q}+3 H \dot{Q}+\frac{d V(Q)}{d Q}+\frac{d V_{I}(Q)}{d Q}=0 .
$$

The source term in the equation above is given by [27]

$$
\frac{d V_{I}(Q)}{d Q}=\sum_{i} n_{i} \frac{d M_{i}}{d Q}\left\langle\frac{M_{i}}{E}\right\rangle=\frac{\beta}{M_{p l}} \frac{1}{\pi^{2}} T \sum_{i} M_{i}^{3} K_{1}\left(M_{i} / T\right),
$$

where $n_{i}$ and $E$ are the number density and energy of the $\mathrm{RH}$ neutrinos respectively, \langle\rangle indicates thermal average. In the last step of the equation we have taken the Maxwell- 

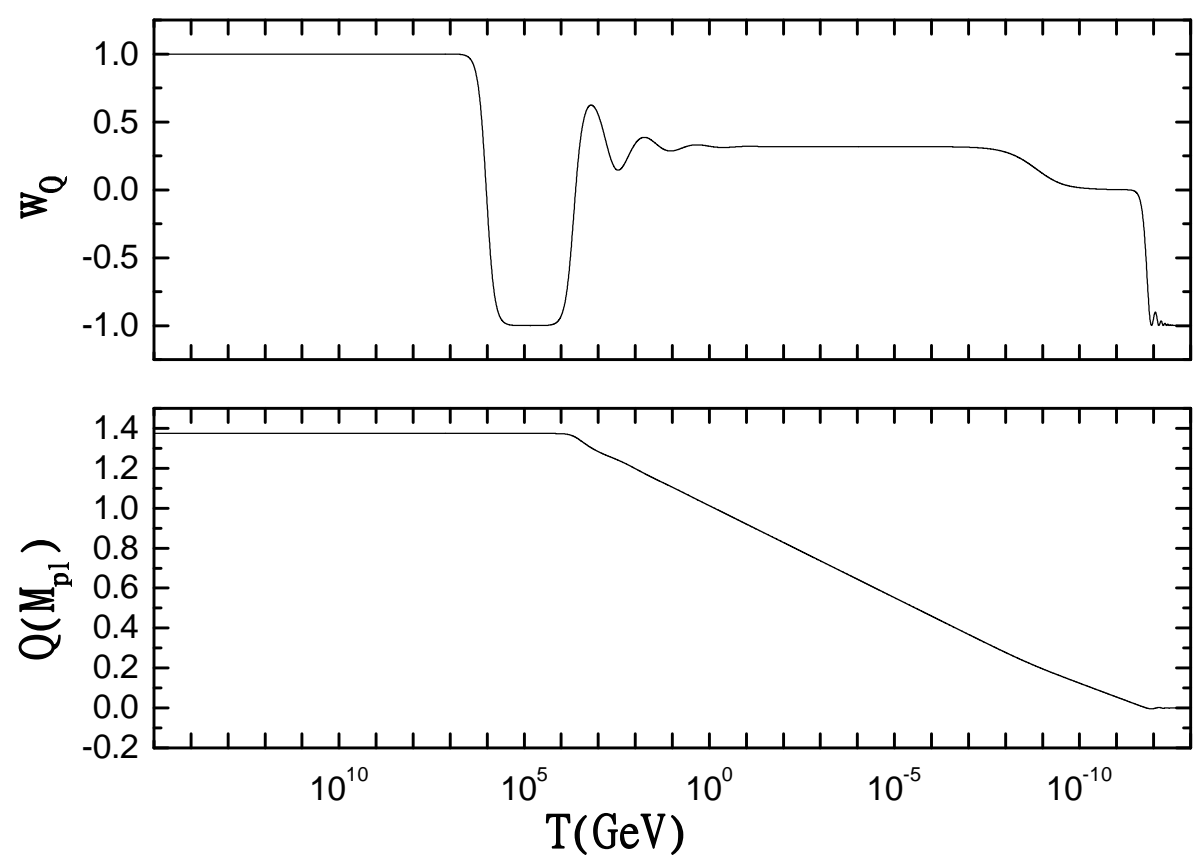

FIG. 6: The evolution of $W_{Q}$ and $Q$ as a function of the temperature $T$ for the double exponential Quintessence model including the coupling with the RH neutrinos. We take $\beta=-2.5$ and $\bar{M}_{1}=$ $3.1 \times 10^{9} \mathrm{GeV}$. We take the same definition of $W_{Q}$ as in Eq. (21).

Boltzmann distribution of the RH neutrinos for simplicity and $K_{1}$ is the modified Bessel function. The energy density of Quintessence is taken the same form as $\rho_{Q}=\dot{Q}^{2} / 2+V(Q)$ in this case.

We then solve the equation (22) numerically, assuming $\bar{M}_{3}=10 \bar{M}_{2}=100 \bar{M}_{1}$. The numerical results are shown in Figs. 6 and [7 In Fig. 6. we take $\bar{M}_{1}=3.1 \times 10^{9} \mathrm{GeV}$, $\beta=-2.5$, which gives rise to $Q_{0} \approx 0$ and $Q_{D}=1.374 M_{p l}$. We then have $K \approx 31$ and $M_{1}^{D} \approx 10^{8} \mathrm{GeV}$, corresponding to the case we considered in the hierarchical neutrino spectrum. In Fig. 7 we choose the parameters $\bar{M}_{1}=3.15 \times 10^{10} \mathrm{GeV}$ and $\beta=3.62$. We find the values of $Q_{0}$ and $Q_{D}$ are almost the same as the above case. We then have $K \approx 0.007$ and $M_{1}^{D} \approx 4.5 \times 10^{12} \mathrm{GeV}$, corresponding to the case that satisfy our requirement for the degenerate neutrinos.

Comparing Figs. 6 and 7 with Fig. [5, one can see that the interaction of Quintessence with the $\mathrm{RH}$ neutrinos does change the early behavior of the Quintessence field $Q$ and its equation of state, however, does not change the tracking properties of this model. Furthermore, the 

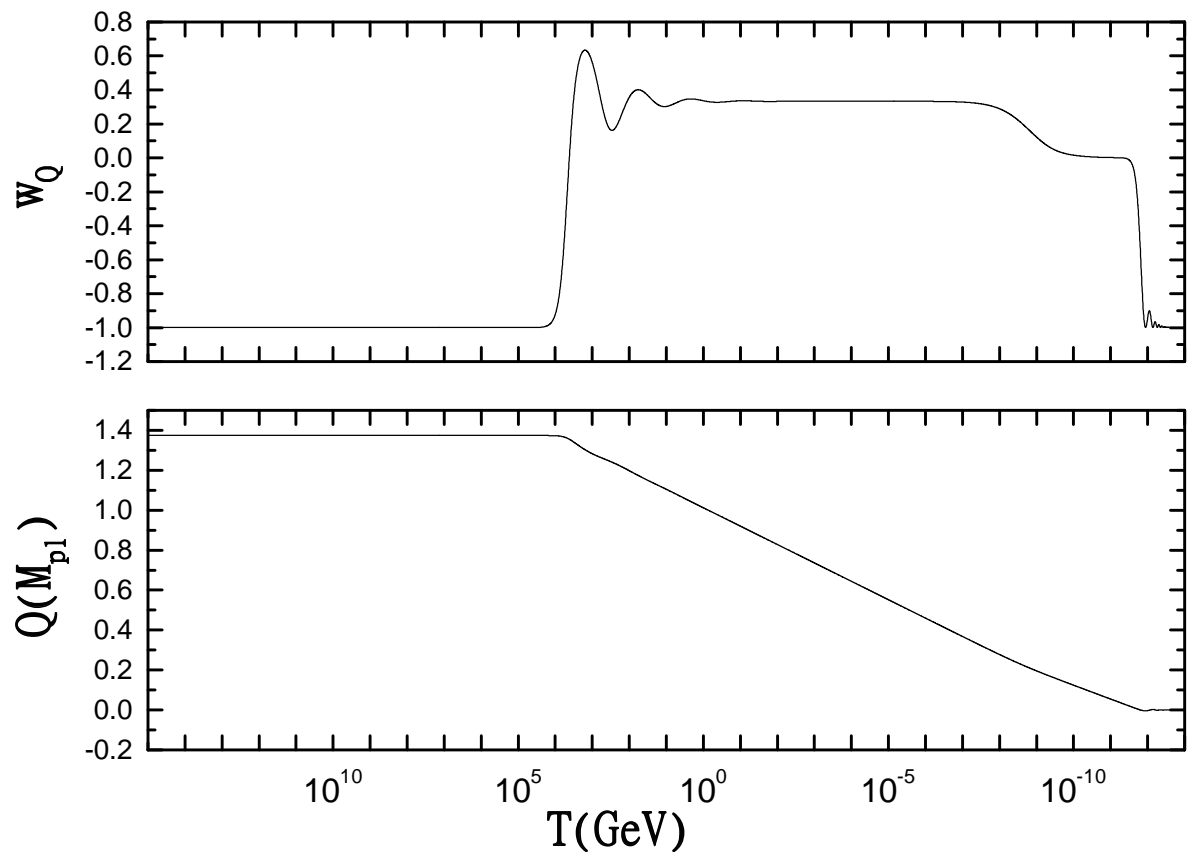

FIG. 7: The evolution of $W_{Q}$ and $Q$ as a function of the temperature $T$ for the double exponential Quintessence model including the coupling with the RH neutrinos. We take $\beta=3.62$ and $\bar{M}_{1}=$ $3.2 \times 10^{10} \mathrm{GeV}$. We take the same definition of $W_{Q}$ as in Eq. (21).

value of Quintessence field $Q$ remains a constant in this model until $T \sim 10^{4} \mathrm{GeV}$ which satisfies our assumption for a constant $K$ during the period of leptogenesis.

\section{SUMMARY AND CONCLUSION}

The explanation of the baryon number asymmetry of the Universe remains a challenge for cosmological and particle physics. Among various theoretical models, leptogenesis is one of the most attractive scenarios. Especially, the minimal thermal leptogenesis only needs the minimal extension of the standard model, while the extension seems necessary in order to explain the neutrino oscillation experiments.

The minimal thermal leptogenesis is closely connected with the properties of the light neutrinos. Detailed studies show that, to generate the observed baryon asymmetry, the constraints $M_{1} \gtrsim 10^{10} \mathrm{GeV}$ and $m_{i} \lesssim 0.1 \mathrm{eV}$ should be satisfied. These results disfavor the degenerate neutrino mass spectrum strongly and require a high reheating temperature. 
We investigate in this paper the implications on the minimal thermal leptogenesis of a new scenario that the RH neutrinos couple with the Quintessence, whose evolution causes the masses of the $\mathrm{RH}$ neutrinos vary. We study the interplay of this coupling between the evolution of Quintessence and the leptogenesis. By solving the Boltzmann equations numerically we show that $M_{1}$, at the decay time, can be as low as about $10^{8} \mathrm{GeV}$ and $m_{i}$, at the present epoch, can be as large as $m_{i} \sim 0.2 \mathrm{eV}$.

\section{Acknowledgments}

We thank B. Feng for helpful discussions about the Quintessence evolution. This work is supported in part by the National Natural Science Foundation of China under the Grand No. 10105004, 19925523, 10047004 and the Ministry of Science and Technology of China

under Grant NO. NKBRSF G19990754. X. J. Bi is also supported in part by the China Postdoctoral Science Foundation.

[1] C. L. Bennett et. al., Astrophys. J. Suppl. 148, 1 (2003), arXiv: astro-ph/0302207; D. N. Spergel et. al., Astrophys. J. Suppl. 148, 175 (2003), arXiv: astro-ph/030209.

[2] For the reviews, see, M. Trodden, arXiv: hep-ph/0302151; V.A. Rubakov, M. E. Shaposhnikov, Usp. Fiz.Nauk 166 (1996) 493; Phys. Usp. 39 (1996) 461; A. G. Cohen, D. B. Kaplan, A. E. Nelson, Ann. Rev. Nucl. Part. Sci. 43 (1993) 27.

[3] M. Fukugita and T. Yanagida, Phys. Lett. B174, 45 (1986).

[4] N. S. Manton, Phys. Rev. D28, 2019 (1983); F. R. Klinkhamer and N. S. Manton, Phys. Rev. D30, 2212 (1984); V. A. Kuzmin, V. A. Rubakov and M. E. Shaposhnikov, Phys. Lett. B155, $36(1985)$.

[5] P. Langacker, R. D. Peccei and T. Yanagida, Mod. Phys. Lett. A1 (1986) 541; M. A. Luty, Phys. Rev. D45 (1992) 455; R. N. Mohapatra and X. Zhang, Phys. Rev. D45 (1992) 5331; K. Enqvist and I. Vilja, Phys. Lett. B299 (1993) 281; A. Acker, H. Kikuchi, E. Ma and U. Sarkar, Phys. Rev. D48 (1993) 5006.

[6] M. Flanz, E. A. Paschos and U. Sarkar, Phys. Lett. B345 (1995) 248; E382 (1996) 447; A. Ganguly, J. C. Parikh, U. Sarkar, Phys. Lett. B385 (1996) 175; M. Flanz, E. A. Paschos, 
U. Sarkar and J. Weiss, Phys. Lett. B389 (1996) 693; A. Pilaftsis, Phys. Rev. D56 (1997) 5431; J. Faridani, S. Lola, P. J. O’Donnell, U. Sarkar, Eur. Phys. J. C7 (1999) 543; J. R Ellis, S. Lola and D. V. Nanopoulos, Phys. Lett. B452 (1999) 87; E. Ma, S. Sarkar, U. Sarkar, Phys. Lett. B458 (1999) 73; M.S. Berger and B. Brahmachari, Phys. Rev. D60 (1999) 073009; A. Pilaftsis, Phys. Rev. D60 (1999) 105023; R. Barbieri, P. Creminelli, A. Strumia and N. Tetradis, A. Pilaftsis, Int. J. of Mod. Phys. A14 (1999) 1811; Nucl. Phys. B575 (2000) 61; P. Adhya, D. R. Chaudhuri, Phys. Rev. D61 (2000) 033002; R. Rangarajan, H. Mishra, Phys. Rev. D61 (2000) 043509; P. Adhya, D. R. Chaudhuri, A. Raychaudhuri, Eur. Phys. J. C19 (2001) 183; A. S. Joshipura, E. A. Paschos and W. Rodejohann, Nucl. Phys. B611 (2001) 227; JHEP 0108 (2001) 029; M. Hirsch, S. F. King, Phys. Rev. D64 (2001) 113005; H. B. Nielsen, Y. Takanishi, Phys. Lett. B507 (2001) 241; Nucl. Phys. B636 (2002) 305; T. Asaka, H. B. Nielsen, Y. Takanishi, Nucl. Phys. B647 (2002) 252; T. Hambye, Nucl. Phys. B633 (2002) 171; G. C. Branco, R. G. Felipe, F. R. Joaquim, M. N. Rebelo, Nucl. Phys. B640 (2002) 202; M. S. Berger, K. Siyeon, Phys. Rev. D65 (2002) 053019; W. Rodejohann, K. R. S. Balaji, Phys. Rev. D65 (2002) 093009; D. Falcone, Phys. Rev. D66 (2002) 053001; J. M. Cline, V. A. Yajnik, S. N. Nayak, M. Rabikumar, Phys. Rev. D66 (2002) 065001; J. Ellis and M. Raidal, Nucl. Phys. B643 (2002) 229; J. R. Ellis, M. Raidal and T. Yanagida, Phys. Lett. B546 (2002) 228; W. Rodejohann, Phys. Lett. B 542 (2002) 100; S. Pascoli, S. T. Petcov and W. Rodejohann, hep-ph/0302054; T. Hambye, Nucl. Phys. B633 (2002) 171; hep-ph/0307237; F. Buccella, D. Falcone and F. Tramontano, Phys. Lett. B524 (2002) 241; T. Endoh, S. Kaneko, S. K. Kang, T. Morozumi, M. Tanimoto, Phys. Rev. Lett. 89 (2002) 231601; A. Broncano, M. B. Gavela and E. Jenkins, Phys. Lett. B552 (2003) 177; M. N. Rebelo, Phys. Rev. D67 (2003) 013008; R. Allahverdi and A. Mazumdar, Phys. Rev. D67 (2003) 023509; G. C. Branco, R. Gonzalez Felipe, F. R. Joaquim, I. Masina, M. N. Rebelo and C. A. Savoy, Phys. Rev. D67 (2003) 073025; L. Boubekeur, S. Davidson, M. Peloso and L. Sorbo, Phys. Rev. D67 (2003) 043515; S. F. King, Phys. Rev. D67 (2003) 113010; K. Hamaguchi, hep-ph/0212305.

[7] S. Davidson, A. Ibarra, Phys. Lett. B535 (2002) 25.

[8] M. Plümacher, Nucl. Phys. B530 (1998) 207; W. Buchmüller and M. Plümacher, Int. J. Mod. Phys. A15 (2000) 5047; W. Buchmüller, P. Di Bari and M. Plümacher, Nucl. Phys. B643 (2002) 367; W. Buchmüller, P. Di Bari, M. Plümacher, Phys. Lett. B 547 (2002) 128; W. Buchmüller, P. Di Bari, M. Plümacher, Nucl. Phys. B 665 (2003) 445. 
[9] Y. Grossman, T. Kashti, Y. Nir, E. Roulet, arXiv: hep-ph/0307081; G. D'Ambrosio, G. F. Giudice, M. Raidal, arXiv: hep-ph/0308031 A. Pilaftsis, T. E. J. Underwood, arXiv: hep-ph/0309342.

[10] G.F. Giudice, A. Notari, M. Raidal, A. Riotto and A. Strumia, arXiv: hep-ph/0310123

[11] M. Gell-Mann, P. Ramond and R. Slansky, Proceedings of the Supergravity Stony Brook Workshop, New York, 1979, eds. P. Van Nieuwenhuizen and D. Freedman (North-Holland, Amsterdam); T. Yanagida, Proceedings of the Workshop on Unified Theories and Baryon Number in the Universe, Tsukuba, Japan 1979 (eds. A. Sawada and A. Sugamoto, KEK Report No. 79-18, Tsukuba); R. N. Mohapatra and G. Senjanovic, Phys. Rev. Lett. 44, 912 (1980); S. L. Glashow, Caraese lectures, (1979).

[12] Y. Fukuda et al (Super-Kamionkande Collaboration), Phys. Rev. Lett. 81 (1998) 1158; Phys. Rev. Lett. 82 (1999) 1810; Q. R. Ahmad et al (SNO collaboration), Phys. Rev. Lett. 89 (2002) 011301; K. Eguchi et al, ( KamLAND Collaboration), Phys. Rev. Lett. 90 (2003) 021802.

[13] J. R. Ellis, J. E. Kim and D. V. Nanopoulos, Phys. Lett. B 145 (1984) 181; J. R. Ellis, D. V. Nanopoulos and S. Sarkar, Nucl. Phys. B 259 (1985) 175; J. R. Ellis, D. V. Nanopoulos, K. A. Olive and S. J. Rey, Astropart. Phys. 4 (1996) 371; M. Kawasaki and T. Moroi, Prog. Theor. Phys. 93 (1995) 879; T. Moroi, Ph.D. thesis, arXiv:hep-ph/9503210 M. Bolz, A. Brandenburg and W. Buchmüller, Nucl. Phys. B 606 (2001) 518; R. Cyburt, J. R. Ellis, B. D. Fields and K. A. Olive, astro-ph/0211258.

[14] H. V. Klapdor-Kleingrothaus, A. Dietz, H. L. Harney, I. V. Krivosheina, Mod. Phys. Lett. A 16 (2001) 2409.

[15] S. W. Allen, R. W. Schmidt and S. L. Bridle, astro-ph/0306386.

[16] S. Pelmutter et al., Astrophys. J. 517, 565 (1998); A. G. Riess et al., Astron. J. 116, 1009 (1998).

[17] M. Tegmark, et al, [the SDSS collaboration], astro-ph/0310723.

[18] S. M. Carroll, Phys. Rev. Lett. 81 (1998) 3067; C. Kolda and D. H. Lyth, Phys. Lett. B 458 (1999) 197; R. Horvat, Mod. Phys. Lett. A 14 (1999) 2245; J. High Energy Phys. 08 (2002) 031; R. D. Peccei, hep-ph/0009030 L. Amendola, Phys. Rev. D 62 (2000) 043511; L. Amendola and D. Tocchini-Valentini, Phys. Rev. D 66 (2002) 043528; P. J. E. Peebles, astro-ph/0208037; M. Gasperini, F. Piazza and G. Veneziano, Phys. Rev. D 65 (2002) 023508; M. Pietroni, Phys. Rev. D67 (2003) 103523. 
[19] J. K. Webb et al., Phys. Rev Lett 87 (2001) 091301.

[20] M. Li, X. Wang, B. Feng and X. Zhang, Phys. Rev. D 65 (2002) 103511; M. Li and X. Zhang, hep-ph/0209093 to appear in Phys. Lett. B ; X. J. Bi, M. Li and X. Zhang, hep-ph/0308218.

[21] P. Gu, X. Wang and X. Zhang, Phys. Rev. D 68 (2003) 087301.

[22] D. Comelli, M. Pietroni and A. Riotto, Phys. Lett. B571 (2003) 115; R. Fardon, A. E. Nelson and N. Weiner, astro-ph/0309800.

[23] R. N. Mohapatra, X. Zhang, Phys. Rev. D 45 (1992) 2699; G. F. Giudice, M. E. Shaposhnikov, Phys. Lett. B 326 (1994) 118.

[24] S. Y. Khlebnikov, M. E. Shaposhnikov, Nucl. Phys. B 308 (1988) 885; J. A. Harvey, M. S. Turner, Phys. Rev. D 42 (1990) 3344.

[25] D. Comelli, M. Pietroni, A. Riotto, Phys. Lett. B 571 (2003) 115.

[26] T. Barreiro, E. J. Copeland, N. J. Nunes, Phys. Rev. D 61 (2000) 127301.

[27] G. R. Farrar and P. J. E. Peebles, astro-ph/0307316 